\title{
Analysis of Electrical Circuit Fault Problems of Electromechanical Equipment
}

\author{
Tao Chen \\ College of mechanical and electrical engineering, Xinjiang Vocational and Technical College of \\ Communications, Urumqi Xinjiang, 831401, China
}

Key words: Electromechanical equipment, Electrical circuit, Fault problem, Analysis .

\begin{abstract}
Under the background of national economic development, the application range of electromechanical equipment becomes wider, and electrification degree also improves. Thus, safety of electrical circuit operation receives much attention. But, in the long operation process of electromechanical equipment, faults are inevitable. Once a fault occurs, maintenance personnel must proficiently master fault maintenance steps of electromechanical equipment, and take feasible measures to electrical circuit fault.
\end{abstract}

\section{Introduction}

As the degree of automation deepens, electromechanical equipment operation situation must be valued to guarantee normal implementation of routine production, which is directly related to social and economic development as well as people' s daily life. In order to achieve normal operation of electromechanical equipment, electric power department and personnel must effectively maintain electromechanical equipment, and regularly detect electrical circuit in an all-round way. Once any circuit fault or potential safety hazard, it is required to analyze the reasons in detail and take actions to handle and solve it in time.

\section{Practical significance of unobstructed electrical circuit of electromechanical equipment}

Electrical equipment includes transformer, different types of power distribution units, standby power device and electrical line etc. However, due to the influence of many factors (especially actual working environment or high voltage and large current, it is necessary to improve equipment requirements $^{[1]}$. However, the circuit itself is very complex, and practical workload of equipment is heavy. Under the influence of human and natural factors, many faults will occur to electrical circuit and then seriously affect normal operation of intelligence system. Thus, it must be maintained in time.

Un-obstruction of electromechanical equipment circuit is closely related to safe operation of intelligence system. Thus, it is required to check the circuit in an all-round way to ensure equipment operation safety. The quality of electromechanical equipment has the direct bearing on construction quality. To guarantee unobstructed circuit, the most important point is that wiring is more reliable ands firmer. Besides, equipment welding quality must be strictly controlled to avoid adverse effect on power supply quality. Once a fault exists, it is necessary to check and maintain the equipment in time, and actively construct inspection and maintenance system so as to achieve normal operation of electrical equipment. On this basis, because transmission voltage upgrades continuously, the requirements for electrical equipment also improve. Once a fault happens, negative effect will inevitably occur to operation safety of architectural intelligence system. Thus, it is required to attach importance to electrical equipment maintenance, and make sure the circuit is unobstructed all the time. This is also the key to sustainable development of building industry. 


\section{Detection and maintenance steps for electrical circuit fault of electromechanical equipment}

For the electromechanical equipment in the operation process, once it goes wrong, equipment maintenance personnel should detect and maintenance it in time. The detailed steps are as follows:

Firstly, maintenance personnel need to proficiently master electromechanical equipment operation and installation steps so that they can stop the equipment in time in case of any fault and find out the specific fault position according to the installation drawings ${ }^{[2]}$. Meanwhile, maintenance personnel need to overall and deeply understand the electrical circuit wiring drawing of electromechanical equipment, including operation principle, and study main maintenance methods and steps for the components which may be damaged easily.

Secondly, if electromechanical equipment goes wrong in the operation process, maintenance personnel should communicate with the operator in time and then comprehensively grasp the situations of the equipment before and after the fault as well as fault results. Such mode can help maintenance personnel accurately find out the fault position in a short time, shorten the time for fault removal and improve maintenance efficiency. This can make sure the enterprise can recover operation in a short time.

Thirdly, after the fault is grasped, maintenance personnel should explore fault position deeply according to electrical circuit and construction drawings of equipment so as to find out the fault position.

Fourthly, after fault position is found out, the optimal maintenance method should be adopted for different fault points. If no problem is found in appearance inspection process, maintenance personnel should detect and maintain the equipment from internal result of fault position and operation principle. Generally, equipment detection and maintenance includes the fault points within and beyond the routine maintenance range. For the fault point within the routine maintenance range, maintenance personnel should handle it in accordance with routine maintenance plan. For the fault point beyond the routine maintenance range, maintenance personnel should analyze fault position and especially operation principle of fault position in detail, and try to find out the reasons in design aspect so as to avoid repeated occurrence of similar problem.

\section{Analysis method for electrical circuit fault of electromechanical equipment}

In the operation of electromechanical equipment, circuit fault is a common open circuit fault. To be more specific, when the electrical equipment operates, the circuit is disconnected due to the fault, which finally influences circulation effect of loop current and results in abnormal operation of electrical element in the electromechanical equipment. At present, the detection methods of open electrical circuit fault include short connection, resistance measurement and voltage measurement ${ }^{[3]}$.

\section{Short connection}

Firstly, local short connection. When the voltage is normal, short connection is conducted for two adjacent mark points. If pull-in situation exists to KM1 of two points during short connection, open circuit exists between the two points. Local short connection is applicable to the situation where there are few components or there is only one fault.

Secondly, segmented short connection. In this method, it is necessary to fix a short connection line at a point, and then move the other end gradually to reduce the times of short connection. If one end of short connection line is fixed at a point, and the other end is moved to No.3 point, the existence of KM1 actuation phenomenon indicates a fault exists between No.3 point and a point. Then, the short line is moved to No.1 point or No.2 point until the specific fault position is found. But, it is unnecessary to measure the segment between No.3 point and No.5 point. If there is no KM1 actuation phenomenon, the fault must exist between No.3 point and No.5 point. Then, it is necessary to detect each segment to finally find out the specific fault position. Segmented short connection method is application to the situation of multiple detections or one fault. 


\section{Resistance measurement}

Firstly, hierarchical measurement. In practical measurement, power supply of circuit needs to be cut off to avoid damage to the multimeter. In addition, it is necessary to disconnect the circuit to be measured and other circuits to avoid influence in other circuits. Especially the parallel circuit with the circuit to be measured may easily influence detection result, thus leading to error in follow-up work. If the measured value is same with or close to the theoretical value, this indicates there is no fault in circuit wiring. But, if the numerical value between the two differs a lot, this indicates poor contact exists in the circuit. If the coil measured is negative and the resistance value is zero, this also shows short circuit exists to the coil.

Secondly, segmented measurement. A common method used to analyze electrical circuit fault of electromechanical equipment is segmented measurement. The naturally open point in the circuit serves as the segment point and is divided into 2-3 segments. First of all, the resistance value of each segment of circuit is measured. If the value is large, this indicates open circuit exists in this segment. Then, this segment of circuit is detected step by step to make sure the fault point can be found out accurately.

\section{Voltage measurement}

Firstly, hierarchical measurement. Such detection method is very practical. The test probe is connected with the multimeter and one end of electrical equipment to be measured. The other end is connected with different points of circuit. Then, the multimeter is used at different points and the fault position is found out. If there is no fault between the multimeter and test probe in the measurement process, the reading of multimeter should be equal to the voltage value of power supply. If voltage reading between the point and the measured point is zero, open circuit exists between the two points. Then, the multimeter is needed to measure the voltage between the two points to correctly confirm open circuit position.

Secondly, segmented measurement. The principles of hierarchical measurement and segmented measurement are basically consistent. Electrical equipment is detected segment by segment. Such measurement method is applicable to circuit detection with a wide range of short circuit, and can effectively save detection time.

Thirdly, short connection. For electromechanical equipment, if the load of fault point is small, short connection method should be used to detect short circuit fault. An intact wire should be prepared. For the possible fault range, short connection is carried out for both ends of faulty circuit. If the circuit is connected under short connection state, this shows a fault exists in this circuit. Then, a uniform method is need to narrow fault range and find out the specific fault position finally.

\section{Case analysis of electrical fault of electromechanical equipment}

\section{Sparking fault of carbon brush and slip ring of generator}

In the operation process of generator, a common electrical fault is carbon brush sparking of slip ring. If it cannot be handled in time, equipment ring fire may be caused easily, and even safety and service life of generator may be affected. The reason for this fault is as follows: the pressure of compressed spring, operation time and quality differ, so the resistance of contact point between carbon brush and slip ring differs. Under the function of uneven current in the slip ring at the same level and different carbon brushes, compressed spring will deform or be damaged ands finally form sparking ${ }^{[4]}$. Besides, the materials of many carbon brushes of electrical generators are disqualified, so abrasion will form due to vibration in carbon box. Even carbon brush edge will fall. Finally, uneven collecting ring abrasion will appear, which will trigger unit vibration, make dust accumulate in brush box and brush carrier and result in sparking of carbon brush. Since the unit inspectors have careless omission in practical work, and fail to discover and handle too high temperature of carbon brush, sparking finally occurs to the carbon brush. 
The specific measures for the above problems are as follows. Firstly, slip ring and compressed spring of generator should be replaced to make the model is uniform. Meanwhile, pressure of compressed spring should be tested according to maintenance situation to make carbon brush is consistent, relative to the pressure of collecting ring. Secondly, if carbon brush quality of generator is disqualified, it is required to replace it in time and make sure the length exceeds $2 / 3$ of that of new carbon brush. But, it's worth noting that the replaced carbon brush shall not exceed 1/5 every time. Thirdly, if the carbon brush of generator is new, it should be ground to make sure actual contact area between carbon brush and surface of slip ring is larger than $70 \%$ of carbon brush area. On this basis, it is required to make sure the carbon brush is within the allowed motion range. Finally, the personnel need to systematically inspect the slip ring, compressed spring and carbon brush etc. of generator to make sure the unit operates normally.

\section{Motor fault}

For high-voltage motor, poor contact of outgoing line may easily influence normal operation of motor. This is also a common electrical circuit fault of electromechanical equipment. The reason is that the operation environment of motor is moist, which causes the insulation is affected with damp. In addition, since the dust is heavy, magnetic substance on coil surface falls off and stator winding insulation is broken down and grounded. Besides, because the lead of motor is in the hot wind area, thermal ageing may easily occur. Due to the influence of external force, the outgoing line of motor goes wrong. For low-voltage motor, a common fault is startup fault. When the motor is connected with power sully, too low voltage of power supply, sealing-off and breakage of cage-type rotor of motor are main reasons for motor startup fault.

The solutions to the above problems are as follows. For stator winding grounding fault caused by high-voltage motor, voltage drop method should be used to find out the fault position. Generally, the rotor is drawn out. After the accurate grounding fault point is found out, repair measures should be taken in time. Besides, local coil of stator winding grounding point should be repaired in time, and the outgoing line in hot wind area should be isolated and eliminated. Then, insulation should be reinforced again. The number of binding ropes should increase between stator winding and junction box to prevent looseness between outgoing line and porcelain insulator. For low-voltage motor startup fault, the specific solution is to measure power supply voltage and adopt methods to improve voltage and make it stable ${ }^{[5]}$. Moreover, it is required to inspect and repair sealing-off and breakage of cage-type rotor in time. Meanwhile, it is required to regularly inspect local coil of rotor and stator and repair it in time.

\section{Short circuit caused by insulating layer}

If the insulating layer of equipment goes wrong, short circuit fault may occur easily and then influence normal operation of electromechanical equipment. The major reason is that the personnel fail to pay attention to electromechanical equipment maintenance. Thus, insulating layer of some equipment is affected with damp or aged. Finally, it falls off or is damaged, and thus short circuit fault happens.

For this fault, the solution is as follows: repair insulating layer fault of electromechanical equipment, handle the damage problem of insulating layer and rationally arrange equipment and line so as to avoid damage to the equipment. In one word, it is required to take anticorrosion, weather-proof or dustproof measures, try to use dual power supply and ensure automatic switchover of power supply.

\section{Conclusion}

In conclusion, electrical circuit fault is most common in the operation process of electromechanical equipment, and it is inevitable. Thus, maintenance personnel need to know the steps and methods of fault detection, take measures to solve faults in time and guarantee quality and efficiency of 
electromechanical equipment operation. Meanwhile, equipment overhaul and maintenance should be valued to reduce fault probability as far as possible.

\section{References:}

[1] Yang Yongquan, Analysis of electrical circuit fault of electromechanical equipment. Construction Materials \& Decoration, 2016(23):227-228.

[2] Wei Na, Analysis of electrical circuit fault of electromechanical equipment and improvement measures. Shandong Industrial Technology, 2016(16):171.

[3] Hu Yiping, On electrical circuit fault of electromechanical equipment and solutions. Science \& Wealth, 2016(8):656-656.

[4] Ding Dawei, Analysis of electrical circuit fault of electromechanical equipment and fault handling. China CIO News, 2013(6):124.

[5] Li Songming, Discussion on electrical circuit fault of electromechanical equipment and fault handling. Construction Materials \& Decoration, 2013(7):124-125. 